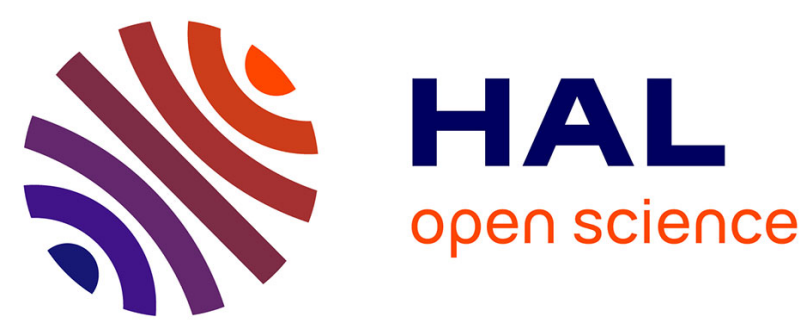

\title{
SPATIALLY UNSUPERVISED ANALYSIS OF WITHIN-SUBJECT FMRI DATA USING MULTIPLE EXTRAPOLATIONS OF 3D ISING FIELD PARTITION FUNCTIONS
}

Thomas Vincent, Laurent Risser, Philippe Ciuciu, J. Idier

\section{To cite this version:}

Thomas Vincent, Laurent Risser, Philippe Ciuciu, J. Idier. SPATIALLY UNSUPERVISED ANALYSIS OF WITHIN-SUBJECT FMRI DATA USING MULTIPLE EXTRAPOLATIONS OF 3D ISING FIELD PARTITION FUNCTIONS. 2009 IEEE INTERNATIONAL WORKSHOP ON MACHINE LEARNING FOR SIGNAL PROCESSING (MLSP 2009), Sep 2009, Grenoble, France. pp.103-108. cea-00470636

\section{HAL Id: cea-00470636 https://hal-cea.archives-ouvertes.fr/cea-00470636}

Submitted on 7 Apr 2010

HAL is a multi-disciplinary open access archive for the deposit and dissemination of scientific research documents, whether they are published or not. The documents may come from teaching and research institutions in France or abroad, or from public or private research centers.
L'archive ouverte pluridisciplinaire HAL, est destinée au dépôt et à la diffusion de documents scientifiques de niveau recherche, publiés ou non, émanant des établissements d'enseignement et de recherche français ou étrangers, des laboratoires publics ou privés. 


\title{
SPATIALLY UNSUPERVISED ANALYSIS OF WITHIN-SUBJECT FMRI DATA USING MULTIPLE EXTRAPOLATIONS OF 3D ISING FIELD PARTITION FUNCTIONS
}

\author{
Thomas Vincent ${ }^{1,2}$, Laurent Risser ${ }^{1,2,3}$, Philippe Ciuciu ${ }^{1,2}$ and Jérôme Idier ${ }^{3}$ \\ ${ }^{1}$ NeuroSpin/CEA, 91191 Gif-sur-Yvette, France \\ 2 IFR 49, Institut d'Imagerie Neurofonctionnelle, Paris, France \\ ${ }^{3}$ IRCCyN/CNRS, 1 rue de la Noë, 44300 Nantes, France \\ ${ }^{1}$ firstname.lastname@cea.fr ${ }^{3}$ firstname.lastname@irccyn.ec-nantes.fr,
}

\begin{abstract}
In this paper, we present a fast numerical scheme to estimate Partition Functions (PF) of symmetric Ising fields. Our strategy is first validated on 2D Ising fields. and then applied to the joint detection-estimation of brain activity from functional Magnetic Resonance Imaging (fMRI) data, where the goal is to automatically recover activated regions and estimate the region-dependent hemodynamic filter. For any region, a specific 3D Ising field may embody spatial correlation over the hidden states of the voxels by modeling whether they are activated or not. To make spatial regularization adaptive, our approach is first based upon a classical path sampling method to approximate a small subset of reference PFs corresponding to prespecified regions. Then, we propose an extrapolation method that allows us to approximate the PFs associated to the Ising fields defined over the remaining brain regions. In comparison with preexisting approaches, our method is robust against grid inhomogeneities within the reference PFs and remains efficient irrespective of the topological configurations of the reference and test regions. Our contribution strongly alleviates the computational cost and makes spatially adaptive regularization of whole brain fMRI datasets feasible.
\end{abstract}

\section{INTRODUCTION}

In medical image analysis, one is often interested in recovering spatial structures. A simple but suboptimal approach to enhance signal-to-noise ratios (SNR) consists in filtering the datasets at the expense of a loss of spatial resolution. A more challenging approach works on the unsmoothed data by introducing some prior knowledge on the sought spatial structures. Spatial information is usually embedded in local interaction models such as Markov Random Fields (MRFs), which depend on a set of hyper-parameters. For instance, the temperature level controls the amount of spatial correlation in symmetric Ising models. In the considered fMRI application [1], which aims at analyzing 4D signals to jointly perform dynamics estimation and activation detection, the MRF definition is

The autors thank the Région île de France for funding. region-specific. Indeed, the Hemodynamic Filter (HF) is assumed to be invariant only locally, so that neuroimaging data is accordingly segmented into $\Gamma$ functionnally homogeneous irregular parcels, $\Gamma$ being the order of several hundreds. This leads to a region-based analysis where $\Gamma$ independent HFs have to be identified. Each model yields a HF shape estimate and spatial mixture models (SMM) are jointly expressed on the amplitude of the HF for every stimulus type to perform activation detection. SMMs in turn imply the involvement of discrete Ising fields to model spatial correlation. Therefore, several hundreds of temperature levels have to be estimated making a hand-tuning procedure unrealistic. Moreover, since optimal setting of such parameter may be different when considering different regions of the brain, all temperature levels cannot be fixed to the same value. The purpose of this paper is then to provide an unsupervised and adaptive regularization scheme in such a situation.

For a single field, unsupervised spatial regularization consists in estimating the temperature level. This requires a precise estimation of the PF that makes the MRF integrable over its domain. Section 2 is dedicated to the formulation of the PF estimation problem for Ising fields. The main contribution of this paper lies in Section 3 where a fast extrapolation technique to PF estimation of 3D Ising Fields is proposed and validated both in the $2 \mathrm{D}$ and $3 \mathrm{D}$ context since the former offers the opportunity to provide a ground truth to the PF computation of Ising fields. The application to Joint Detection Estimation (JDE) of brain activity in fMRI is presented in Section 4. Conclusions are drawn in Section 5.

\section{PROBLEM STATEMENT}

Let us consider a grid characterized by a set of sites $\mathbf{s}=$ $\left(s_{i}\right)_{i=1: n}$. A binary label $q_{i} \in\{0,1\}$ is associated to each site $s_{i}$. A pair of adjacent sites $s_{i}$ and $s_{j}(i \neq j)$ is denoted $i \sim j$ and is called a clique $c$. The set of all cliques allows us to define an undirected graph denoted $\mathcal{G}$. Let $\boldsymbol{q}=$ $\left(q_{1}, q_{2}, \cdots, q_{n}\right) \in\{0,1\}^{n}$ be the set of binary labels associated to $s$. In what follows, we assume $\boldsymbol{q}$ to be distributed 
according to a symmetric Ising model:

$$
\operatorname{Pr}(\boldsymbol{q} \mid \beta)=Z(\beta)^{-1} \exp (\beta U(\boldsymbol{q})),
$$

where $U(\boldsymbol{q})=\sum_{i \sim j} I\left(q_{i}=q_{j}\right)$ is the global "negative energy" and $I(A)=1$ whenever $A$ is true and 0 otherwise. The inverse temperature $\beta \geq 0$ controls the amount of spatial correlation between the components of $\boldsymbol{q}$ according to $\mathcal{G}$. The partition function $Z(\beta)$ reads $\sum_{\boldsymbol{q} \in\{0,1\}^{n}} \exp (\beta U(\boldsymbol{q}))$ and depends on the geometry of $\mathcal{G}$. Its exact evaluation in a reasonable amount of time is impossible except on tiny grids. Robust and fast estimation of $Z(\beta)$ is thus a key issue for numerous 3D medical imaging problems involving Ising models and more generally discrete MRFs.

\section{PARTITION FUNCTION ESTIMATION}

Several approaches have been designed to estimate a single PF [2-4]. Path-sampling is an extension of importance sampling for estimating ratios of normalizing constants, by considering series of easy-to-sample unormalized intermediate densities. Such a strategy was proven efficient to tabulate the PF for the Ising case; see [5] for details. Algorithms with polynomial time complexity $[4,6]$ provide efficient alternatives to a single PF estimation. However, none of them is able to perform multiple PF estimation at the same time. Since several hundreds of grids of variable size and shape are manipulated in our fMRI application, fast estimation of multiple $\mathrm{PF}$ is necessary. To this end, we propose a hybrid scheme which consists in resorting to path sampling to get log-scale estimates $\left(\log \widehat{Z}_{\mathcal{G}_{p}}(\beta)\right)_{p=1: P}$ in a small subset of reference graphs $\left(\mathcal{G}_{p}\right)_{p=1: P}$ and then in using extrapolation formulas to obtain $\log \widetilde{Z}_{\mathcal{T}}(\beta)$ for the large remaining set of brain regions to be analyzed, referenced here by a test graph $\mathcal{T}$.

\subsection{Linear/bilinear regression schemes}

In [5], the authors have proposed a linear regression procedure to estimate $\left(\log \widehat{Z}_{\mathcal{G}_{p}}(\beta)\right)_{p=1: P}$ as a function of the number of cliques in the grids $\left(\mathcal{G}_{p}\right)_{p=1: P}$. Estimates of $\log \widetilde{Z}_{\mathcal{T}}(\beta)$ are then linearly computed using the estimated regression coefficients and the number of cliques in $\mathcal{T}: \log \widetilde{Z}_{\mathcal{T}}\left(\beta_{k}\right)=$ $\widehat{a}_{k} c_{\mathcal{T}}$ at each $\beta_{k}$ regularization level $\left(\beta_{k}=k \Delta \beta\right)$.

A bilinear extension of this technique, which also takes the number of sites in the grid into account, has been developed in [7]. This procedure was shown to be efficient to estimate log-PFs in small and irregular grids ${ }^{1}$ such as those appearing in our fMRI application. However, the accuracy of linear/bilinear PF extrapolations strongly depends on the homogeneity and the number of reference grids: the less homogeneous the reference set, the larger the approximation error. These reasons motivate the development of a more reliable and versatile approach.

\footnotetext{
${ }^{1}$ Here, by irregular grids we make reference to regular lattic es combined with non-straight boundaries.
}

\subsection{Fast and robust extrapolation technique}

Our algorithm proceeds in two steps: 1) Akin to [5], reference PFs $\widehat{Z}_{\mathcal{G}_{p}}\left(\beta_{k}\right)$ are estimated using path sampling. The topological configurations of the reference grids $\left(\mathcal{G}_{p}\right)_{p=1: P}$ can be inhomogeneous to cover a maximum of situations that may occur when dealing with a brain parcellation into functionally homogeneous ROIs. 2) For any test grid $\mathcal{T}$, the quantity $\log Z_{\mathcal{T}}$ is approximated from a single reference $\log$-PF estimate out of $\left(\log \widehat{Z}_{\mathcal{G}_{p}}(\beta)\right)_{p=1: P}$ selected by an appropriate criterion. Let $n_{i}$ be the number of neighbors for site $s_{i}$ and $r_{\mathcal{T}}=\sigma_{n, \mathcal{T}} / \mu_{n, \mathcal{T}}$ a measure of grid homogeneity where $\mu_{n, \mathcal{T}}$ and $\sigma_{n, \mathcal{T}}$ provide the average number of neighbors per site over $\mathcal{T}$ and the corresponding standard deviation, respectively: the smaller $r_{\mathcal{T}}$ the more regular $\mathcal{T}$. Our topological similarity measure given by $\mathcal{L}_{\mathcal{T}}\left(\mathcal{G}_{p}\right)=\left\|r_{\mathcal{T}}-r_{\mathcal{G}_{p}}\right\|^{2}$ helps us choosing the closest reference grid $\mathcal{G}_{\text {ref }}$ to $\mathcal{T}$ in combination with the approximation error criterion $\mathcal{A}_{\mathcal{T}}\left(\beta, \mathcal{G}_{p}\right)$ defined by:

$\mathcal{A}_{\mathcal{T}}\left(\beta, \mathcal{G}_{p}\right)=\left\|\log Z_{\mathcal{T}}(\beta)-\log \widetilde{Z}_{\mathcal{T}}\left(\beta, \mathcal{G}_{p}\right)\right\|^{2} /\left\|\log Z_{\mathcal{T}}(\beta)\right\|^{2}$

with $\log \widetilde{Z}_{\mathcal{T}}\left(\beta, \mathcal{G}_{p}\right)=\left(\frac{c_{\mathcal{T}}}{c_{\mathcal{G}_{p}}}\left(\log \widehat{Z}_{\mathcal{G}_{p}}(\beta)-\log 2\right)+\log 2\right)$,

where $\left(c_{\mathcal{T}}, c_{\mathcal{G}_{p}}\right)$ and $\left(n_{\mathcal{T}}, n_{\mathcal{G}_{p}}\right)$ are the number of cliques and sites of the Ising fields defined over $\mathcal{T}$ and $\mathcal{G}_{p}$, respectively. Our extrapolation formula (2) is built up according to two principles: i.) an unbiased asymptotic approximation error ${ }^{2}$ and $i$.) an exact approximation of $\left(\log Z_{\mathcal{T}}(\beta)\right)^{\prime}$ for $\beta \rightarrow 0^{+}$. These principles are summarized in Appendix A. The reference grid $\mathcal{G}_{\text {ref }}$ is exhibited using a min-max principle, which consists in minimizing with respect to (wrt) all reference grids $\left(\mathcal{G}_{p}\right)_{p=1: P}$ the maximal approximation error $\mathcal{A}\left(\beta, \mathcal{G}_{p}\right)$. In Appendix $\mathrm{B}$, it is shown that $\mathcal{A}\left(0, \mathcal{G}_{p}\right)=\max _{\beta} \mathcal{A}\left(\beta, \mathcal{G}_{p}\right), \forall \mathcal{G}_{p}$. Hence, we get:

$$
\begin{aligned}
& \mathcal{G}_{\text {ref }}=\underset{\left(\mathcal{G}_{p}\right)_{p=1: P}}{\arg \min } \mathcal{A}_{\mathcal{T}}\left(0, \mathcal{G}_{p}\right) \quad \text { subject to } \quad \mathcal{L}_{\mathcal{T}}\left(\mathcal{G}_{p}\right) \leq \epsilon \\
& \text { and } \mathcal{A}_{\mathcal{T}}\left(0, \mathcal{G}_{p}\right) \triangleq\left\|\left(n_{\mathcal{T}}-1\right)-c_{\mathcal{T}}\left(n_{\mathcal{G}_{p}}-1\right) / c_{\mathcal{G}_{p}}\right\|^{2} / n_{\mathcal{T}}^{2}
\end{aligned}
$$

where $\epsilon>0$ is a positive threshold fixed by hand. Once $\mathcal{G}_{\text {ref }}$ has been identified, the $\log$-PF estimate in $\mathcal{T}$ is thus given by $\log \widetilde{Z}_{\mathcal{T}}\left(\beta, \mathcal{G}_{\text {ref }}\right)$ according to Eq. (2).

Our method is illustrated in Fig. 1 with $P=4$ by comparing the distance between the reference log-PFs with the test one at $\beta=0$. It appears that $\log \widehat{Z}_{\text {ref }}$ is the closest curve above the ground truth $\log \widehat{Z}_{\mathcal{T}}$ (in red) and that our log-PF estimate $\log \widetilde{Z}_{\mathcal{T}}$ represented by crosses $(\times)$ is superimposed on the path sampled curve.

\subsection{Assesment of the method}

\subsection{1. $2 D$ fields}

We first need to validate our approach in a situation for which the $\log \mathrm{PF}$ admits a closed form expression. Surprisingly, this

\footnotetext{
${ }^{2} \lim _{\beta \rightarrow+\infty} \mathcal{A}_{\mathcal{T}}\left(\beta, \mathcal{G}_{p}\right)=0$.
} 


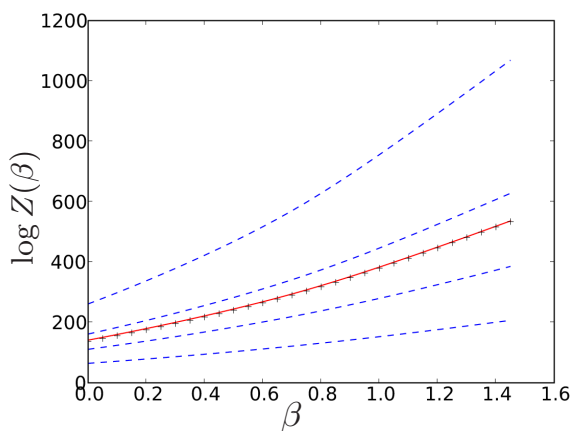

Fig. 1. Path sampled estimates of the reference log-PFs $\log \widehat{Z}_{\mathcal{G}_{p}}\left(\beta_{k}\right), p=1: 4$, in blue curves. Ground truth as the log-PF estimate found by path sampling $\log \widehat{Z}_{\mathcal{T}}$ in red. Our extrapolation method provides the crossed-line $(\times-) \log$-PF estimate $\log \widetilde{Z}_{\mathcal{T}}$

situation exists thanks to the contribution of Onsager [8], who derived the closed form expression of the log-PF of any 2D square Ising fields under toroidal boundary constraints:

$$
\log Z(\beta)=n(\beta+\log [2 \cosh \beta]+\psi[u(\beta)])
$$

where $u(\beta)=2 \sinh \beta / \cosh ^{2} \beta$ and the $\psi$ function is a one dimensional log-elliptic integral:

$$
\psi(u)=\frac{1}{2 \pi} \int_{0}^{2 \pi} \log \left[\left(1+\sqrt{1-u^{2} \sin ^{2} x}\right) / 2\right] d x
$$

for $u \in[0,1]$. Therefore, the huge summation in Eq. (1) is equivalent to this far simpler one dimensional equation.

In Fig. 2, we compared the exact calculation provided by Eq. (5) with the path-sampling and extrapolation approaches on a $2 \mathrm{D}$ Ising field defined over a $30 \times 30$ regular grid. It is shown that our extrapolation technique (red) is as accurate as possible since our estimate is superimposed on the ground truth (in blue). Moreover, it appears on Fig. 2 that these numerical approaches slightly underestimate the true log-FP as given by Eqs (5). We have checked that this small discrepancy is independent of the grid size. It might be due to the use of the Swendsen-Wang sampling scheme for correlated fields.

\subsection{2. $3 D$ fields}

For validation purpose, we compared log-PF estimates computed using our extrapolation technique with those obtained using path sampling, considered as the ground truth. Reference and test graphs are either regular or irregular. A total of 15 regular reference large (more than $10^{3}$ sites) graphs are considered with cubic, planar and curvilinear shapes. Irregular graphs were extracted from regular bounding boxes in which Ising field configurations were drawn using the temperature dependent Swendesen-Wang algorithm [9]. In each bounding box, we considered the largest connected component of sites having the same label as an irregular graph. Irregular reference graphs were then computed using 170 bound-

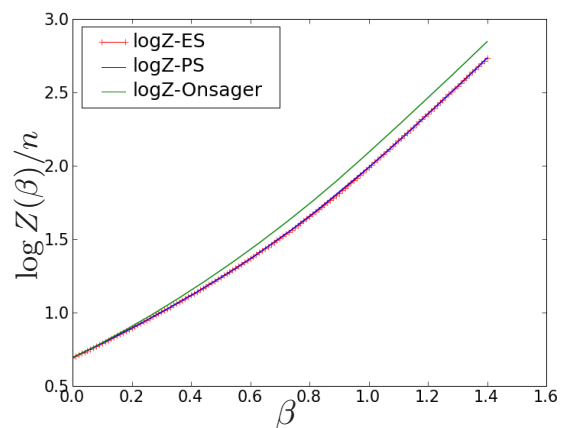

Fig. 2. Green: True $\log Z(\beta)$ computed by Eqs. (5)-(6) for a $2 \mathrm{D}$ Ising field defined over a 30x30 regular grid. Blue: corresponding path-sampling estimate i.e., $\log \widehat{Z}(\beta)$. Red: Extrapolation estimates $\log \widetilde{Z}(\beta)$ from a reference set made up by 30 grids (1D, 2D and 3D).

ing boxes of increasing size (from $10^{3}$ to $15^{3}$ sites) and regularization levels $\beta$ within the range $[0.2,0.7]$. Regular test graphs form three subsets: 30 of them are small (less than $10^{3}$ sites), 30 are medium size (between $10^{3}$ and $15^{3}$ sites) and 30 are large (more than $15^{3}$ sites). Finally, irregular test graphs also form three subsets. Each contain 30 graphs obtained from bounding boxes of $16^{3}$ sites, for $\beta=0.2,0.4$ and 0.5 , respectively. We compared then our extrapolation method with the alternative proposed in [5] and its bilinear extension developed in [7]. Percentages of the mean maximal approximation errors are presented in Table 1.

The bilinear and extrapolation methods clearly outperform the linear one. Moreover, as shown in col. (B/ R) and rows (regular small and irregular, $\beta=0.2$ ), the bilinear method leads to inaccurate estimates when there are strong topological differences between the reference and test grids. The regular reference grids are actually composed of large grids with cubic, planar and curvilinar shapes whereas those lying in (regular small) and (irregular $\beta=0.2$ ) are very small and highly sparse. In that case, our extrapolation method detects such differences and still succeeds in providing reliable log-PF estimates. While the linear/bilinear methods take all reference grids into consideration to derive a log-PF approximation, our approach computes a log-PF estimate using the most appropriate reference grid. Hence, the larger the set of reference grids the more accurate our extrapolation method becomes. This explains why the reference subsets are successfully mixed in the proposed approach, as shown in the first column of Table 1. Interestingly, when both the reference and test grids are irregular the bilinear method may provide a competitive alternative to our extrapolation technique.

\subsection{A Monte Carlo study to hyper-parameter estimation}

The last validation we examined addresses the estimation of the inverse temperature level (i.e., $\beta$-estimation) in the Maximum Likelihood (ML) sense either from our log-PF estimate or from its path sampled counterpart. This study has been 
Table 1. Mean maximal approximation error over regular and irregular test graphs. Both linear, bilinear and extrapolation techniques are tested. Errors are given in percents.

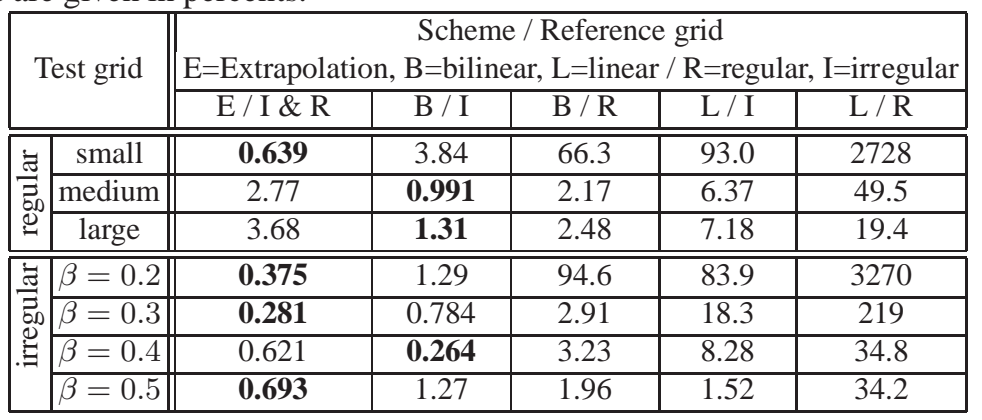

conducted directly on observed 3D Ising fields. At each temperature level $\beta_{k}=k \Delta \beta$ with $\Delta \beta=.1$, we generated independently 100 3D Ising fields defined over the same parallelepipedical grid. We tested different grid sizes (from $10^{3}$ to $50^{3}$ ) and showed that the number of voxels only influences the error bars on the $\beta$ estimate. For an Ising field defined by Eq. (1), the ML estimate $\widehat{\beta}^{\mathrm{ML}}$ is given by $\widehat{\beta}^{\mathrm{ML}}=$ $\arg \max _{\beta}[\beta U(\boldsymbol{q})-Z(\beta)]$. In Fig. 3, we compared two ML estimators corresponding to the path sampling and extrapolation method for estimating the log-PFs. As illustrated in Fig. 3, our extrapolation technique (red curve) retrieves the true regularization parameter for $\beta<0.7$. For $0.7<\beta<1$, a very small bias is observed while for lor larger values, a more significant error occurs in comparison to a more precise path-sampling scheme (blue curve).

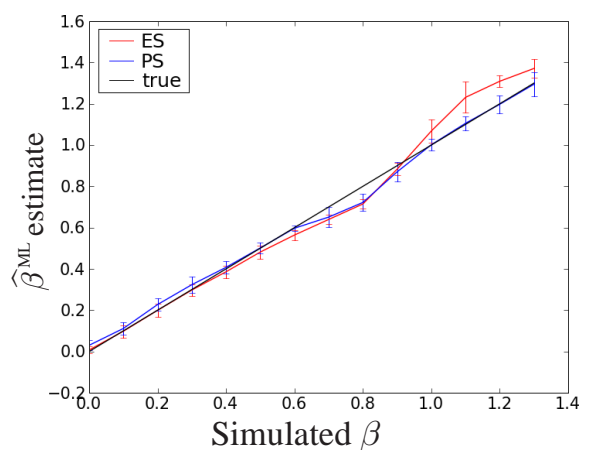

Fig. 3. Monte Carlo validation (100 realizations) for $\beta$-estimation

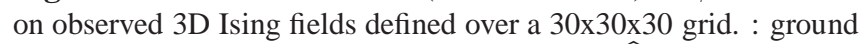
truth given by the first bisector. : ML estimate $\widehat{\beta}^{\mathrm{ML}}$ relying on our log-PF extrapolation method. : ML path-sampled estimate $\beta^{\mathrm{ML}}$.

\section{APPLICATION TO FMRI DATA ANALYSIS}

\subsection{Problem statement}

Our extrapolation algorithm was applied to the spatially adaptative regularization of the region-based Joint Detection-Estimation (JDE) of brain activity introduced in [1]. The JDE approach relies on a prior parcellation of the brain into $\mathcal{P}=\left(\mathcal{P}_{\gamma}\right)_{\gamma=1: \Gamma}$ functionally homogeneous and connected parcels [10]. Every parcel $\mathcal{P}_{\gamma}$ comprising voxels $\left(V_{j}\right)_{j=1: J}$ is characterized by a single HRF $\boldsymbol{h}$. Within a given $\mathcal{P}_{\gamma}$, voxel-dependent and stimulus-related fluctuations of the BOLD signal magnitude are encoded by $\mathrm{a}=\left(a_{j}^{m}\right)_{j=1: J, m=1: M}$, the response levels ( $m$ stands for the stimulus type index). The fMRI time course measured in voxel $V_{j}$ then reads: $\boldsymbol{y}_{j}=\sum_{m=1}^{M} a_{j}^{m} \boldsymbol{x}^{m} \star \boldsymbol{h}+$ $\boldsymbol{b}_{j}$, where $\boldsymbol{x}^{m}$ stands for the $m^{\text {th }}$ binary stimuli vector and $\boldsymbol{b}_{j}$ stands for the noise component [1]. Within the Bayesian framework, prior probability density functions (pdfs) are introduced on $(a, h)$ [1]. Spatial Gaussian mixture models are expressed on a through the introduction of hidden variables $\mathbb{q}=\left(\boldsymbol{q}_{j}^{m}\right)_{j=1: J}^{m=1: M}$ that encode whether voxel $V_{j}$ is activating in response to stimulus $m\left(q_{j}^{m}=1\right)$ or not $\left(q_{j}^{m}=0\right)$. Hence, stimulus-dependent hidden Ising fields are introduced on these states such that the global prior pdf reads:

$$
p\left(\mathrm{a} \mid \Theta_{\mathrm{a}}\right)=\prod_{m} \sum_{\boldsymbol{q}^{m}}\left[\left(\prod_{j} f\left(a_{j}^{m} \mid q_{j}^{m}, \boldsymbol{\theta}_{m}\right)\right) \operatorname{Pr}\left(\boldsymbol{q}^{m} \mid \beta_{m}\right)\right]
$$

and $f\left(a_{j}^{m} \mid q_{j}^{m}=i\right) \sim \mathcal{N}\left(\mu_{i, m}, v_{i, m}\right)$. Parameters $\mu_{i, m}$ and $v_{i, m}$ define the prior mean and variance of class $i=0,1$, respectively for the stimulus type $m$. The set $\boldsymbol{\theta}_{m}$ comprises four prior mixture parameters $\boldsymbol{\theta}_{m}=\left\{\mu_{0, m}, \mu_{1, m}, v_{0, m}, v_{1, m}, \beta_{m}\right\}$. Samples of the full posterior pdf $p(\boldsymbol{h}, \mathrm{a}, \mathbb{q}, \boldsymbol{\Theta} \mid \mathbb{y})$ are simulated using a Gibbs sampler algorithm and posterior mean estimates are then computed from these samples. Here, we introduce the sampling of parameter $\beta_{m}$, which is achieved using a symmetric random walk Metropolis-Hasting step: At iteration $k$, a candidate $\beta_{m}^{(k+1 / 2)} \sim \mathcal{N}\left(\beta_{m}^{(k)}, \sigma_{\epsilon}^{2}\right)$ is generated and is accepted (i.e., $\beta_{m}^{(k+1)}=\beta_{m}^{(k+1 / 2)}$ ) with probability: $\alpha\left(\beta_{m}^{(k)} \rightarrow \beta_{m}^{(k+1 / 2)}\right)=\min \left(1, A_{k, k+1 / 2}\right)$, where the acceptation ratio $A_{k, k+1 / 2}$ follows from Eq. (1):

$$
\begin{aligned}
A_{k, k+1 / 2} & =\frac{p\left(\beta_{m}^{(k+1 / 2)} \mid \boldsymbol{q}_{m}^{(k)}\right)}{p\left(\beta_{m}^{(k)} \mid \boldsymbol{q}_{m}^{(k)}\right)}=\frac{p\left(\boldsymbol{q}_{m}^{(k)} \mid \beta_{m}^{(k+1 / 2)}\right) p\left(\beta_{m}^{(k+1 / 2)}\right)}{p\left(\boldsymbol{q}_{m}^{(k)} \mid \beta_{m}^{(k)}\right) p\left(\beta_{m}^{(k)}\right)} \\
& =\frac{Z\left(\beta_{m}^{(k)}\right)}{Z\left(\beta_{m}^{(k+1 / 2)}\right)} \exp \left(\left(\beta_{m}^{(k+1 / 2)}-\beta_{m}^{(k)}\right) U\left(\boldsymbol{q}_{m}^{(k)}\right)\right),
\end{aligned}
$$

using Bayes' rule and considering a uniform prior for $\beta_{m}$. The $\beta_{m}$ sampling step then requires to estimate ratios of $Z($.) 
or log-PF differences for all $\mathcal{P}_{\gamma}$ parcels prior to exploring the full posterior pdf.

\subsection{Results on real fMRI data}

We applied the JDE procedure to real fMRI data recorded during an experiment designed to map auditory, visual and motor brain functions, which consisted of a single session of $N=125$ scans lasting TR $=2.4 \mathrm{~s}$ each, yielding $3-\mathrm{D}$ volumes composed of $64 \times 64 \times 32$ voxels. The paradigm was a fast event-related design comprising sixty auditory, visual and motor stimuli, declined in 10 experimental conditions (auditory phrase, visual phrase, left auditory or visual clic ...).

We compare three versions of the JDE procedure: Independent Mixture Models (IMM), Supervised SMM (SSMM, $\beta=0.8$ ) and unsupervised SMM (USMM), in order to assess the impact of the adaptive spatial correlation model. Fig. 4 shows normalized contrast maps $\left(\widehat{\boldsymbol{a}}^{\mathrm{LAC}}-\widehat{\boldsymbol{a}}^{\mathrm{RAC}}\right)$ of auditory induced left versus right clic (LAC vs. RAC). As expected, the activations lie in the contralateral right motor cortex. Here, only USMM is more sensitive illustrating thus the advantage of an adaptive spatial correlation model. Indeed, $\widehat{\beta}^{\mathrm{PM}}$ estimates with USMM for the left auditory clic was 0.56 so that the supervised setting of SSMM with $\beta=0.8$ leads to too much correlation and less sensitive results. Interestingly, Fig. 4 also depicts the parcel-dependent maps of the PM $\widehat{\beta}$ estimates for the RAC and LAC experimental conditions. The gain in sensitivity in the USMM contrast map ( $\widehat{\boldsymbol{a}}^{\mathrm{LAC}}-\widehat{\boldsymbol{a}}^{\mathrm{RAC}}$ ) results from a difference in the amount of spatial regularization introduced between the two conditions involved in the contrast. A lower regularization level is estimated $\left(\widehat{\beta}^{\mathrm{LAC}} \approx\right.$ 0.5 vs. $\widehat{\beta}^{\mathrm{RAC}} \approx 0.75$ ) in parcels located in the right motor cortex since the BOLD signal is stronger for the LAC than for the RAC condition in these regions.

On these real fMRI data, our extrapolation scheme provides log-PFs estimate for a brain parcellation $\left(\mathcal{P}_{\gamma}\right)_{\gamma=1: 300}$ and $\left(\mathcal{G}_{p}\right)_{p=1: 50}$ reference grids. In terms of computational complexity, these log-PF estimates were computed in about ten seconds, a very appealing approach in comparison to path sampling, which requires about one hour for estimating all log-PF estimates for a negligable gain in accuracy (less than $3 \%$ ). Finally, we did not observe any significance difference between the USSM effect maps derived using path sampling and our extrapolation scheme (results not shown).

\section{CONCLUSION}

In order to make spatially adaptive regularization feasible, the considered joint detection-estimation of brain activity from unsmoothed fMRI data requires a reliable and fast estimation of 3D Ising field partition function. To this end, an extrapolation algorithm that exploits pre-computed path-sampled logPF estimates on reference grids has been proposed. The approximation error is controlled so that the approach defaults to the robust path-sampled PF estimates if no suitable reference candidate is found. Obviously, efficiency is conditionned by the number of reference grids, and more importantly by their similarity to the topologies encountered in the conducted analysis. In practice, about ten problem-specific reference grids are enough to provide good PF estimates.

Using our fast extrapolation technique, the computational burden remains acceptable since whole brain data analysis at the subject level takes about $1 \mathrm{~h} 30$. The application to real fMRI data showed a gain in statistical sensitivity for the unsupervised version. In order to test their reproducibility, these promising subject-level results have to be confirmed in group studies. Finally, in order to address the estimation of putative deactivations such as those occuring in epilepsy, a direct extension of the proposed methodology to three-class Potts fields is currently investigated.

\section{REFERENCES}

[1] T. Vincent, P. Ciuciu, and J. Idier, "Spatial mixture modelling for the joint detection-estimation of brain activity in fMRI," in 32th Proc. IEEE ICASSP, Honolulu, Hawaii, Apr. 2007, vol. I, pp. 325-328.

[2] X.L. Meng and W.H. Wong, "Simulating ratios of normalizing constants via a simple identity: a theoretical exploration," Stat Sin, vol. 6, pp. 831-860, 1996.

[3] A. Gelman and X.-L. Meng, "Simulating normalizing constants: from importance sampling to bridge sampling path sampling," Stat Sci, vol. 13, pp. 163-, 1998.

[4] M. Jerrum and A. Sinclair, "Polynomial-time approximation algorithms for the Ising model," SIAM J. Comput., vol. 22, pp. 1087-1116, 1993.

[5] Adrien Trillon, Jérôme Idier, and Pierre Peureux, "Unsupervised Bayesian 3D reconstruction for nondestructive evaluation using gammagraphy," in $E U$ SIPCO, Lausanne, Suisse, Aug. 2008.

[6] F. Forbes and N. Peyrard, "Hidden Markov Random Field model selection criteria based on mean field-like approximations," IEEE PAMI, vol. 25, pp. 1089-, 2003.

[7] L. Risser, J. Idier, and P. Ciuciu, "Bilinear extrapolation scheme for fast estimation of $3 \mathrm{D}$ ising field partition function. Application to fMRI time course analysis.," submitted to Proc. IEEE ICIP, Feb. 2009.

[8] L. Onsager, "A two-dimensional model with an orderdisorder transition," Phys. Rev., vol. 65, no. 3\& 4, pp. 117-149, Feb. 1944.

[9] David M. Higdon, James E. Bowsher, Valen E. Johnson, Timothy G. Turkington, David R. Gilland, and Ronald J. Jaszczak, "Fully Bayesian estimation of Gibbs hyperparameters for emission computed tomography data," IEEE Trans. Med. Imag., vol. 16, pp. 516-526, 1997. 

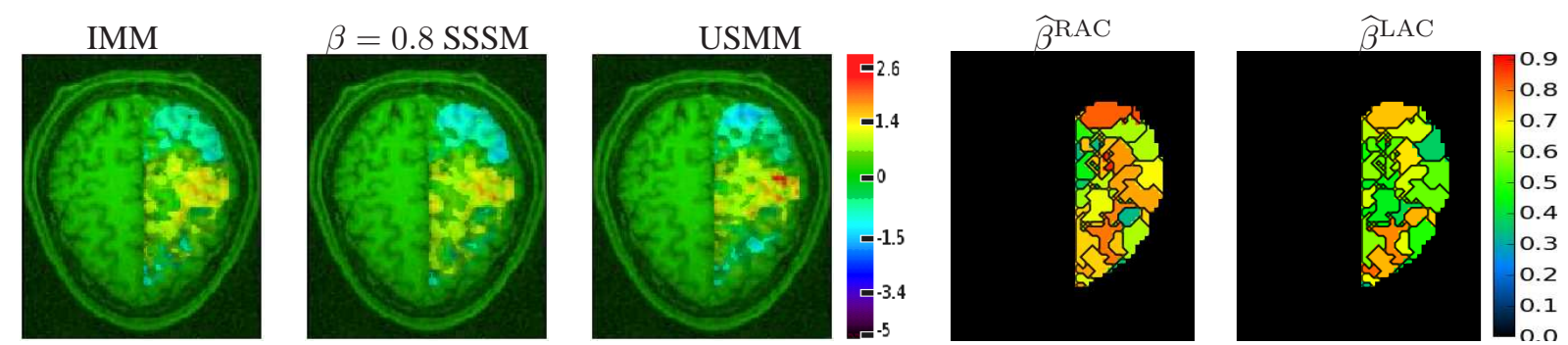

Fig. 4. Bottom: Comparison of the IMM, SSMM and USMM models wrt the estimated normalized constrat maps: left auditory clic (LAC) versus right auditory clic (RAC): $\widehat{\boldsymbol{a}}^{\mathrm{LAC}}-\widehat{\boldsymbol{a}}^{\mathrm{RAC}} . \widehat{\beta}$ parcel-dependent maps computed for the LAC and RAC conditions.

[10] B. Thirion, G. Flandin, P. Pinel, A. Roche, P. Ciuciu, and J.-B. Poline, "Dealing with the shortcomings of spatial normalization: Multi-subject parcellation of fMRI datasets," Hum. Brain Mapp., vol. 27, pp. 678-, 2006.

\section{A. PROPERTIES OF OUR LOG-PF ESTIMATE}

The first property deals with the asymptotic behavior $(\beta \rightarrow$ $\infty)$ of the log-PF:

$$
\lim _{\beta \rightarrow \infty}(\log Z(\beta)-\beta c)=\log 2 .
$$

It is quite straightforward to demonstrate that when $\beta \rightarrow$ $\infty$ only homogeneous configurations of $\boldsymbol{q}$ have a significant weight in the evaluation of $Z(\beta)$. For a symmetric Ising field, such configurations arise whenever all sites are equal to 0 or to 1 leading first to $\sum_{k \sim j} I\left(q_{j k}=q_{j}\right)=c$ and finally to Eq. (7). Applying Eq. (7) to the extrapolation context allows one to derive the following proposition.

Proposition $1 \lim _{\beta \rightarrow \infty} \mathcal{A}_{\mathcal{T}}\left(\beta, \mathcal{G}_{p}\right)=0$, so $\log \widetilde{Z}_{\mathcal{T}}\left(\beta, \mathcal{G}_{p}\right) d e$ fined in Eq. (2) provides an asymptotically unbiased estimate of $\log Z_{\mathcal{T}}(\beta), \forall \mathcal{G}_{p}$.

Proof: First, applying Eq. (7) to $\mathcal{G}_{p}$ and using Eq. (2), we get:

$$
\begin{gathered}
\lim _{\beta \rightarrow \infty} \frac{c_{\mathcal{T}}}{c_{\mathcal{G}_{p}}}\left[\log Z_{\mathcal{G}_{p}}(\beta)-\beta c_{\mathcal{G}_{p}}\right]=\frac{c_{\mathcal{T}}}{c_{\mathcal{G}_{p}}} \log 2 \\
\Leftrightarrow \lim _{\beta \rightarrow \infty}\left[\frac{c_{\mathcal{T}}}{c_{\mathcal{G}_{p}}}\left(\log Z_{\mathcal{G}_{p}}(\beta)-\log 2\right)-\beta c_{\mathcal{T}}\right]=0 \\
\Leftrightarrow \lim _{\beta \rightarrow \infty}\left[\log \widetilde{Z}_{\mathcal{T}}\left(\beta, \mathcal{G}_{p}\right)-\beta c_{\mathcal{T}}\right]=\log 2
\end{gathered}
$$

Applying Eq. (7) to $\log Z_{\mathcal{T}}(\beta)$, we obtain $\lim _{\beta \rightarrow \infty}\left[\log Z_{\mathcal{T}}(\beta)\right.$ $\left.\log \widetilde{Z}_{\mathcal{G}_{p}}\left(\beta, \mathcal{G}_{p}\right)\right]=0 \forall \mathcal{G}_{p}$.

The second property gives us the expression of the firstorder derivative of the log-PF at $\beta=0$. On the one hand, following [9], it can be shown that $(\log Z(\beta))^{\prime}=\mathbb{E}[U(\boldsymbol{q}) \mid \beta]$. On the other hand, for $\beta=0$, all sites are independent and follow a uniform Bernoulli distribution. Hence, for each clique $j \sim k$ the two homogeneous configurations $\left(q_{j}, q_{k}\right)=(0,0)$ and $\left(q_{j}, q_{k}\right)=(1,1)$ contribute to $U$ with the same weight of $1 / 4$. We therefore obtain $\mathbb{E}(U(\boldsymbol{q}) \mid \beta=0)=\sum_{k \sim j} 1 / 2$. Finally, by equating the two expressions, we get:

$$
(\log Z(0))^{\prime} \triangleq d \log Z(\beta) /\left.d \beta\right|_{\beta=0}=c / 2 .
$$

From Eq. (2), we get $\left(\log \widetilde{Z}_{\mathcal{T}}\left(\beta, \mathcal{G}_{p}\right)\right)^{\prime}=\frac{c_{\mathcal{T}}}{c_{\mathcal{G}_{p}}}\left(\log Z_{\mathcal{G}_{p}}(\beta)\right)^{\prime}$, hence Eq. (8) allows us to derive that $\forall \mathcal{G}_{p},\left(\log \widetilde{Z}_{\mathcal{T}}\left(0, \mathcal{G}_{p}\right)\right)^{\prime}$ provides an unbiased estimate of $\left(\log Z_{\mathcal{T}}(0)\right)^{\prime}$.

\section{B. MAXIMAL APPROXIMATION ERROR}

We give here a sufficient condition involving that the approximation errors $\mathcal{A}_{\mathcal{T}}\left(\beta, \mathcal{G}_{p}\right)$ of Ising fields defined over $\mathcal{T}$ and $\mathcal{G}_{p}$ achieve their largest value at $\beta=0$.

Proposition $2 \forall \mathcal{G}_{p}$, if $\left(s_{\mathcal{T}}-1\right) / c_{\mathcal{T}} \neq\left(s_{\mathcal{G}_{p}}-1\right) / c_{\mathcal{G}_{p}}$ (Hyp. 1) and $\mathbb{E}_{\mathcal{T}}(U(\boldsymbol{q}) \mid \beta) / c_{\mathcal{T}} \neq \mathbb{E}_{\mathcal{G}_{p}}(U(\boldsymbol{q}) \mid \beta) / c_{\mathcal{G}_{p}}, \forall \beta>0$ (Hyp. 2) then $\mathcal{A}_{\mathcal{T}}\left(0, \mathcal{G}_{p}\right)=\max _{\beta \in \mathbb{R}_{+}} \mathcal{A}_{\mathcal{T}}\left(\beta, \mathcal{G}_{p}\right)$, which expression is given by Eq. (4).

Proof:Let $\operatorname{err}_{\mathcal{T}}\left(\beta, \mathcal{G}_{p}\right)$ be the unnormalized approximation error: $\mathcal{E}_{\mathcal{T}}\left(\beta, \mathcal{G}_{p}\right)=\left(\log Z_{\mathcal{T}}(\beta)-\log \widetilde{Z}_{\mathcal{T}}\left(\beta, \mathcal{G}_{p}\right)\right)^{2}$. We prove that $\mathcal{E}_{\mathcal{T}}\left(0, \mathcal{G}_{p}\right)=\max _{\beta \in R_{+}} \operatorname{err}_{\mathcal{T}}\left(\beta, \mathcal{G}_{p}\right)$ by showing that $\mathcal{E}_{\mathcal{T}}\left(\beta, \mathcal{G}_{p}\right)$ is a strictly decreasing function on $\mathbb{R}_{+}$:

$$
\begin{aligned}
\frac{d \mathcal{E}_{\mathcal{T}}\left(\beta, \mathcal{G}_{p}\right)}{d \beta} & =\underbrace{2\left(\log Z_{\mathcal{T}}(\beta)-\frac{c_{\mathcal{T}}}{c_{\mathcal{G}_{p}}}\left(\log Z_{\mathcal{G}_{p}}(\beta)-\log 2\right)-\log 2\right)}_{f_{1}(\beta)} \\
& \times \underbrace{\left(\mathbb{E}_{\mathcal{T}}(U \mid \beta)-\frac{c_{\mathcal{T}}}{c_{\mathcal{G}_{p}}} \mathbb{E}_{\mathcal{G}_{p}}(U \mid \beta)\right)}_{f_{2}(\beta)}
\end{aligned}
$$

$\mathcal{E}_{\mathcal{T}}\left(\beta, \mathcal{G}_{p}\right)$ is strictly monotonous on $\mathbb{R}_{+}$if $f_{1,2}(\beta) \neq 0 \forall \beta>$ 0 . According to the second hypothesis, we directly obtain $f_{2}(\beta) \neq 0$. Moreover, it is easy to notice that $f_{1}(\beta)=$ $\pm \sqrt{\mathcal{E}_{\mathcal{T}}\left(\beta, \mathcal{G}_{p}\right)}$. Hence, $f_{1}(0) \neq 0$ according to Hyp. 1 and $\lim _{\beta \rightarrow \infty} f_{1}(\beta)=0$ by definition of $\mathcal{E}_{\mathcal{T}}\left(\beta, \mathcal{G}_{p}\right)$. Furthermore, according to the value of $(\log Z(\beta))^{\prime}$ and Hyp. 2, we get: $f_{1}^{\prime}(\beta)=f_{2}(\beta) \neq 0, \forall \beta>0$. Function $f_{1}$ being continue, its sign is then contant over $\mathbb{R}_{+}$and then $f_{1}(\beta) \neq 0, \forall \beta>0$. As a consequence, $\mathcal{E}_{\mathcal{T}}\left(\beta, \mathcal{G}_{p}\right)$ is then stricly monotonous for $\beta>0$. According to Hyp. 1 , we obtain $\mathcal{E}_{\mathcal{T}}\left(0, \mathcal{G}_{p}\right)>0$. Since by definition $\lim _{\beta \rightarrow \infty} \mathcal{E}_{\mathcal{T}}\left(\beta, \mathcal{G}_{p}\right)=0$, function $\mathcal{E}_{\mathcal{T}}\left(\beta, \mathcal{G}_{p}\right)$ is therefore strictly decreasing on $\mathbb{R}_{+}$and finally $\mathcal{E}_{\mathcal{T}}\left(0, \mathcal{G}_{p}\right)=$ $\max _{\beta \in \mathbb{R}_{+}} \mathcal{E}_{\mathcal{T}}\left(\beta, \mathcal{G}_{p}\right)$. Since $\log Z(\beta)$ is a strictly increasing function of $\beta$, its inverse is strictly decreasing on $\mathbb{R}_{+}$, so $\mathcal{A}_{\mathcal{T}}\left(0, \mathcal{G}_{p}\right)=\max _{\beta \in \mathbb{R}_{+}} \mathcal{A}_{\mathcal{T}}\left(\beta, \mathcal{G}_{p}\right)$. 